\title{
Differences in DNA methylation profile of Th1 and Th2 cytokine genes are associated with tolerance acquisition in children with lgE-mediated cow's milk allergy
}

Roberto Berni Canani ${ }^{1,2,4^{*}}$, Lorella Paparo ${ }^{1}$, Rita Nocerino ${ }^{1,4}$, Linda Cosenza ${ }^{1}$, Vincenza Pezzella ${ }^{1}$, Margherita Di Costanzo ${ }^{1}$, Mario Capasso ${ }^{3,4}$, Valentina Del Monaco ${ }^{3,4}$, Valeria D'Argenio $0^{3,4}$, Luigi Greco ${ }^{1,2}$ and Francesco Salvatore ${ }^{3,4}$

\begin{abstract}
Background: Epigenetic changes in DNA methylation could regulate the expression of several allergy-related genes. We investigated whether tolerance acquisition in children with immunoglobulin E (lgE)-mediated cow's milk allergy (CMA) is characterized by a specific DNA methylation profile of Th2 (IL-4, IL-5) and Th1 (IL-10, IFN- - )-associated cytokine genes.

Results: DNA methylation of CpGs in the promoting regions of genes from peripheral blood mononuclear cells and serum level of IL-4, IL-5, IL-10 and INF- $\gamma$ were assessed in children with active IgE-mediated CMA (group 1), in children who acquired tolerance to cow's milk proteins (group 2) and in healthy children (group 3). Forty children (24 boys, aged 3 to 18 months) were enrolled: 10 in group 1, 20 in group 2, and 10 in the control group. The DNA methylation profiles clearly separated active CMA patients from healthy controls. We observed an opposite pattern comparing subjects with active IgE-mediated CMA with healthy controls and group 2 children who outgrew CMA. The IL-4 and IL-5 DNA methylation was significantly lower, and IL-10 and INF- $\gamma$ DNA methylation was higher in active IgE-mediated CMA patients. Gene promoter DNA methylation rates of all cytokines and respective serum levels were strongly correlated. Formula selection significantly influenced cytokine DNA methylation profiles in group 2.

Conclusions: Tolerance acquisition in children with IgE-mediated CMA is characterized by a distinct Th1 and Th2 cytokine gene DNA methylation pattern. These results suggest that DNA methylation may be a target for CMA prevention and treatment.
\end{abstract}

Keywords: Epigenetics, Interleukin-4, Interleukin-5, Interleukin-10, Interferon-ү, Food allergy, Extensively hydrolyzed casein formula, Lactobacillus rhamnosus GG, Hypoallergenic formulae

\section{Background}

Cow's milk allergy (CMA) is one of the most common food allergies (FA) in early childhood, with an estimated incidence ranging between $2 \%$ and $3 \%$ [1]. Evidence suggests that the natural history of CMA is changing, with an increasing persistence until later ages [2]. It has been demonstrated that immunoglobulin E (IgE)-mediated

\footnotetext{
*Correspondence: berni@unina.it

'Department of Translational Medical Science, University of Naples 'Federico

II', Via S. Pansini, 580131 Naples, Italy

${ }^{2}$ European Laboratory for the Investigation of Food-Induced Diseases,

University of Naples 'Federico II', Via S. Pansini, 580131 Naples, Italy

Full list of author information is available at the end of the article
}

CMA could be the first manifestation of the so-called 'atopic march', characterized by the occurrence of other allergic disorders in the years after the onset of CMA [3]. The pathogenetic basis of IgE-mediated CMA is not completely understood. Critical to the development of IgE-mediated chronic allergic inflammation is an increased production of Th2 cytokines, such as interleukins IL-4 and IL-5, which are not adequately counter-regulated by Th1 cytokines, such as interferon gamma (IFN- $\gamma$ ) and IL-10 [4]. We previously found an opposite response profile to these cytokines in freshly isolated peripheral blood mononuclear cells (PBMCs) between children with active 
IgE-mediated CMA and children who outgrew CMA [5]. Evidence suggests that CMA is mediated by a combination of genetic and environmental risk factors. A key mechanism that governs how environmental exposures modulate gene expression is DNA methylation of the cytosine of CpG dinucleotides in the gene-promoter regions, a well characterized epigenetic mechanism [6]. Therefore, the mechanism by which epigenetics contributes to CMA is a critical issue [7]. Epigenetics is the study of heritable changes in transcriptional activity that are not caused by changes in DNA sequence $[8,9]$. Epigenetic marks, such as DNA methylation, seem to work with other components of the cellular regulatory machinery to control the level of genes expression, and several allergy-related genes have been found to be susceptible to epigenetic regulation, including genes important for T-effector pathways, namely IFN- $\gamma$ and IL-4 $[7,10]$. Preliminary data showed that epigenetic modifications at the Th2 locus could be responsible for the establishment, maintenance, and inheritance of the Th2 phenotype, while altered epigenetic regulation is potentially important for the exaggerated Th2 response seen in atopic diseases [11]. Decreased DNA methylation and an increased association with activating histone marks conjointly establish and maintain the euchromatin structure at the Th2 locus of Th2 cells, thereby allowing recruitment of the transcriptional machinery to this region for a rapid and coordinated expression of the Th2-related cytokines [12-14]. Hypermethylation of IFN- $\gamma$ has been positively correlated with pro-allergic IgE production [15]. As DNA methylation is a reversible mechanism and can be changed during disease course [16-19], we evaluated whether tolerance acquisition in children with IgEmediated CMA is characterized by a specific DNA methylation profile of cytokine genes associated with Th2 (IL-4, IL-5) and Th1 (IL-10, IFN- $\gamma$ ) response.

\section{Results \\ Study subjects}

From 3 December 2012 to 30 April 2014, 13 consecutive children with a recent evidence of IgE-mediated CMA were evaluated. All these patients presented with suspected CMA-related signs and symptoms within 5 weeks of study enrollment. Three were excluded because a negative oral food challenge. The ten remaining patients (group 1) were positive at the double blind placebo controlled food challenge (DBPCFC) and reacted between at doses 3 and $10 \mathrm{ml}$. During the same period, 20 subjects with a previous diagnosis of IgE-mediated CMA, but with recent evidence of oral tolerance acquisition demonstrated by a negative DBPCFC were also enrolled in the study (group 2). All subjects were able to consume at least one full cup daily of cow's milk without symptoms. Group 2 patients, received for a median of 384 days (IQR 7.75) one of the following substitutive formulae: extensively hydrolyzed casein formula (EHCF, $n=3$ ); extensively hydrolyzed casein formula containing the probiotic Lactobacillus rhamnosus GG (EHCF + LGG, $n=10$ ); hydrolyzed rice formula (RHF, $n=1$ ); soy formula (SF, $n=3$ ); amino acidbased formula (AAF, $n=3$ ). During the same period, ten healthy children were evaluated and their parents gave informed consent to the study. The main demographic and clinical characteristics of the study population are detailed in Table 1.

\section{Cytokines DNA methylation profiles}

Figure 1 shows the promoter DNA methylation profiles of IL-4, IL-5, IL-10, and INF- $\gamma$ from PBMCs in the three study groups. The DNA methylation profiles clearly separated active CMA patients from healthy controls. We observed an opposite pattern between children with active IgE-mediated CMA and healthy controls. The DNA methylation of IL-4 and IL-5 was lower and of IL-10 and IFN- $\gamma$ was higher in active CMA patients than in healthy controls (Figure 1A, B, C, D). DNA methylation analysis of these cytokine genes clearly also separated CMA patients by disease-state. Subjects with recent evidence of oral tolerance acquisition presented a different methylation profile if compared to active CMA patients. This profile was similar but not identical to that observed in healthy controls (Figure 1A, B, C, D). The backward stepwise Firth regression analysis showed that the best predictor of active CMA was the IL- 5 methylation rate (healthy controls vs active CMA: coefficient -0.151 , SE 0.058; $P<0.0001$; active CMA vs patients who outgrew CMA: coefficient 0.5419, SE 0.2518; $P<0.0001$ ).

We analyzed, through a linear regression analysis, potential factors (age, sex, serum IgE, specific serum IgE anti-epitopes, symptoms, formulae) able to influence IL-5 DNA methylation rate in subjects who outgrew CMA. The variable that most affected the DNA methylation rate of cytokines was the use of the EHCF + LGG formula (coefficient -17.761 , SE 3.86, $P=0.004)$. Accordingly, patients who outgrew CMA after EHCF + LGG treatment presented a different cytokine methylation pattern compared to children treated with other formulae (Figure 2).

\section{Cytokine serum levels}

We evaluated whether DNA methylation in the promoter region negatively correlated with the serum concentration of IL-4, IL-5, IL-10, and INF- $\gamma$ of the three study groups. The DNA methylation rate of all cytokines significantly correlates with the respective serum concentration (Figure 3A, B, C, D). Active IgE-mediated CMA patients showed significantly higher IL-4 and IL-5 and significantly lower IL-10 and INF- $\gamma$ serum levels compared to healthy controls (Figure 4A, B, C, D). Subjects with a recent evidence of oral tolerance acquisition 
Table 1 Main demographic and clinical characteristics of the study population

\begin{tabular}{|c|c|c|c|}
\hline & $\begin{array}{l}\text { Subjects with CMA at diagnosis } \\
\text { Group } 1\end{array}$ & $\begin{array}{l}\text { Subjects outgrown CMA } \\
\text { Group } 2\end{array}$ & $\begin{array}{l}\text { Healthy subjects } \\
\text { Group } 3\end{array}$ \\
\hline$N$ & 10 & 20 & 10 \\
\hline Male, $n(\%)$ & $7(70)$ & $13(65)$ & $4(40)$ \\
\hline Age, m (SD) & $5.5(0.7)$ & $16.9(0.9)$ & $9(4)$ \\
\hline Body weight, kg (SD) & $7.385(964.7)$ & $12.160(720.9)$ & $8.075(3193.5)$ \\
\hline Spontaneous delivery, $n(\%)$ & $5(50)$ & $5(25)$ & $4(40)$ \\
\hline Breastfeeding, $\leq 8$ weeks, $n$ (\%) & $10(100)$ & $20(100)$ & $10(100)$ \\
\hline \multicolumn{4}{|l|}{ Symptoms at the CMA onset } \\
\hline Gastrointestinal, n (\%) & $4(40)$ & $8(40)$ & - \\
\hline Cutaneous, $n(\%)$ & $8(80)$ & $15(75)$ & - \\
\hline Respiratory, $n(\%)$ & $1(10)$ & $6(30)$ & - \\
\hline Total serum IgE, kU/I (SD) & $260.6(230.9)$ & $193.1(209.5)$ & $0.2(0.1)$ \\
\hline nBos d 4, kUA/I (SD) & $6(11.2)$ & $1.9(3)$ & - \\
\hline nBos d 5, kUA/l (SD) & $4.5(7.1)$ & $2(3)$ & - \\
\hline nBos d 6, kUA/l (SD) & $5.4(8.7)$ & $1.4(2.5)$ & - \\
\hline nBos d 8, kUA/l (SD) & $22.7(39.2)$ & $0.9(1.9)$ & - \\
\hline Lactoferrin, kUA/I (SD) & $2(6.1)$ & 0 & - \\
\hline
\end{tabular}

presented a different profile compared to active CMA patients. This profile was similar to that observed in healthy controls (Figure 4).

\section{Discussion}

We found that oral tolerance acquisition in children with IgE-mediated CMA is characterized by an epigenetic regulation involving Th1 and Th2 cytokine genes. The rate of DNA methylation in Th1 and Th2 cytokine gene promoters in PBMCs differed significantly between active CMA children and children who outgrew CMA. The two groups showed an inverse DNA methylation pattern of the IL-4, IL-5, IL-10, and IFN- $\gamma$ genes. Moreover, a strong correlation between gene promoter DNA methylation rates of all cytokines was observed with the respective serum levels. The link between DNA methylation and gene expression has been explored in in vitro studies [20,21]. In a study of $\mathrm{CD}^{+} \mathrm{T}$ lymphocytes of patients with asthma, stimulated with allergens, there was a significant correlation between the degree of methylation and IL-4 concentration [22]. Lovinsky-Desir et al. found a negative correlation between the DNA methylation rate of the IFN- $\gamma$ promoter in CD4 + T lymphocytes and the relative IFN- $\gamma$ gene expression in a cohort of children and adults with allergic asthma [23]; a similar negative correlation between IFN- $\gamma$ DNA methylation and gene expression of $\mathrm{T}$ effector cells was reported by Kohli et al. in children exposed to secondhand smoke and air pollution [24]. Our study is the first to compare methylation status of Th1 and Th2 cytokine genes in children affected by IgE-mediated CMA at different disease stage. Martino et al. retrospectively examined genome-wide DNA methylation profiles in $\mathrm{CD} 4^{+} \mathrm{T}$ cells from a birth cohort of children with IgEmediated food allergy and age-matched non-allergic controls at birth and at age 12 months [25]. They found dysregulation of DNA methylation of MAPK signaling-associated genes during early $\mathrm{CD} 4^{+} \mathrm{T}$ cell development. They suggested that this may contribute to a suboptimal T-lymphocyte response in early childhood potentially associated to the development of food allergy. The use of epigenetic biomarkers, mainly based on DNA methylation, is well established for the diagnosis and prognosis of tumors and has begun to be understood in other conditions such as autoimmune diseases [26]. The identification of DNA methylation marks in immune-function-related genes could encourage the development of new epigenetic-basedbiomarkers that could have direct application in food allergy. DNA methylation level of selected Th1 and Th2 cytokines genes could be used as a potential biomarker in predicting tolerance acquisition. In this view, Syed et al. showed that the FOXP3 DNA methylation rate decreased during successful oral immunotherapy in patients with peanut allergy and remain stable when oral tolerance is achieved [27]. In accordance with these data, our results suggest that in IgE-mediated CMA children, the DNA methylation of the Th1 and Th2 cytokine genes is a dynamic process with substantial differences occurring during the disease course. The magnitude of 


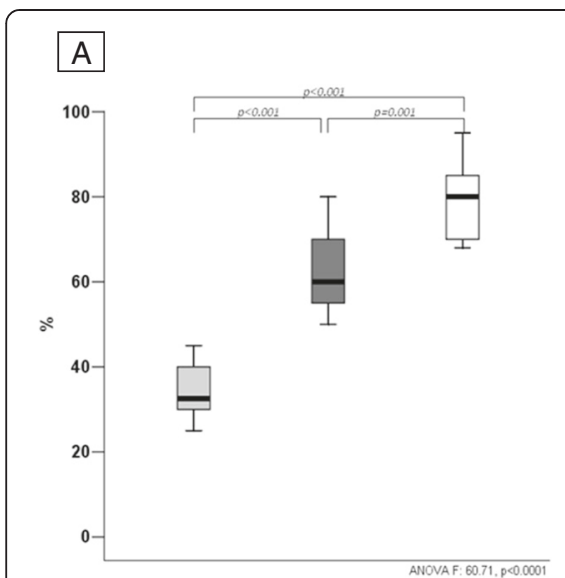

C

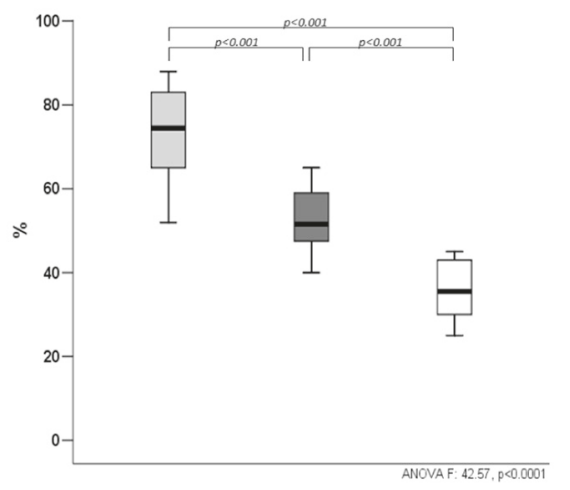

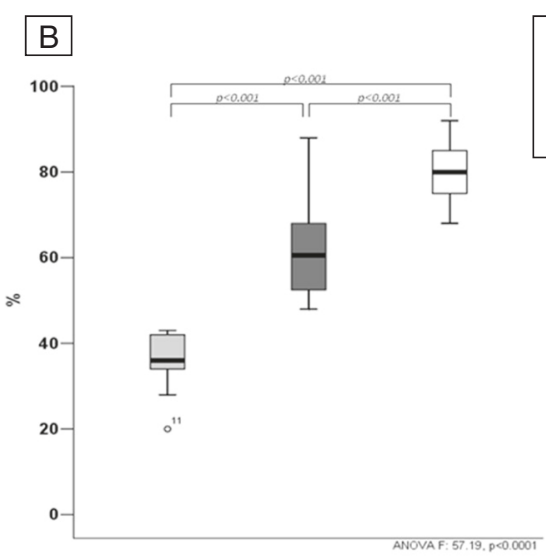

D

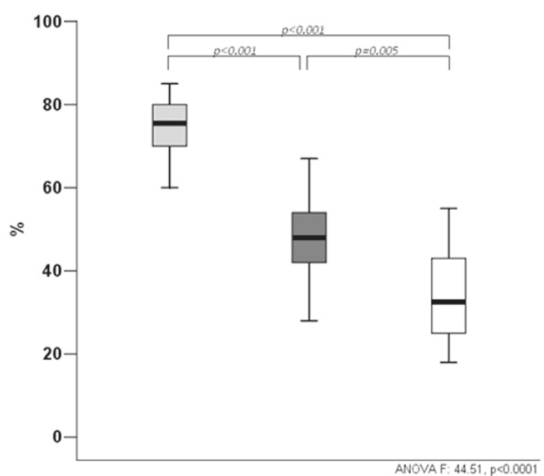

The p-values remain significant after Bonferroni correction

Figure 1 DNA methylation rate of the IL-4, IL-5, IL-10, and INF- $\gamma$ genes (A, B, C, D, respectively) observed in the study population. The rate of promoter gene methylation of IL-4 and IL-5 was lower and IL-10 and IFN- $\gamma$ was higher in group 1 than group 3. Group 2 presented a methylation rate similar to that observed in group 3. (group 3 vs group 1: coefficient -0.151 , SE 0.058; $P<0.0001$; group 1 vs group 2: coefficient 0.542 , SE 0.2518; $P<0.0001)$.

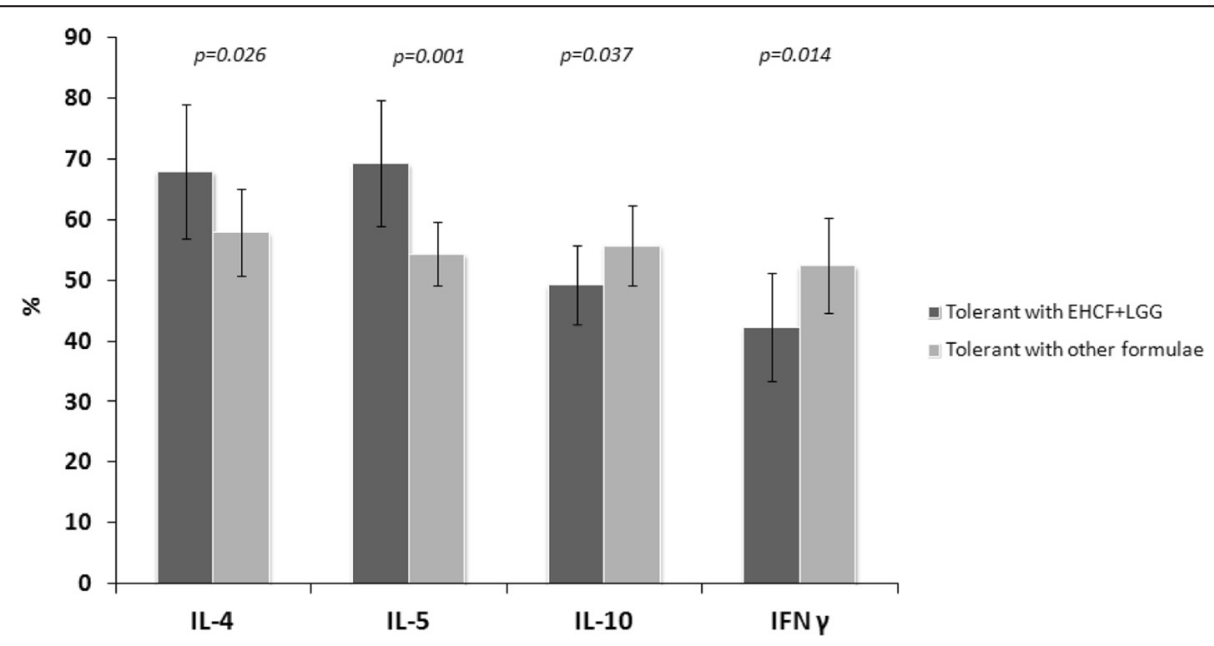

Figure 2 Different DNA methylation rates of the IL-4, IL-5, IL-10, INF- $\gamma$ genes in children who outgrew CMA receiving different dietary treatment. Children, who outgrew CMA after EHCF + LGG treatment, presented a different cytokines methylation profile compared to children treated with other formulae. 


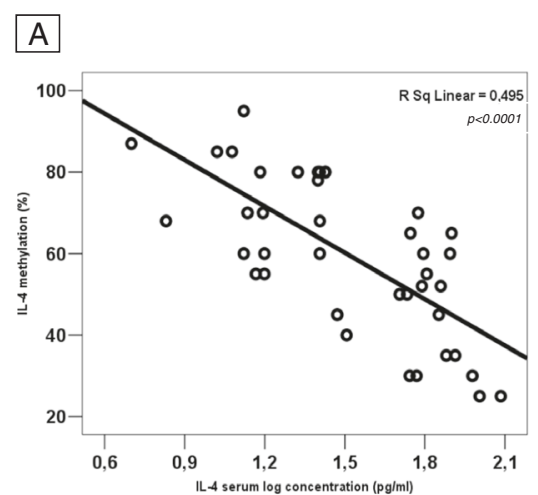

C

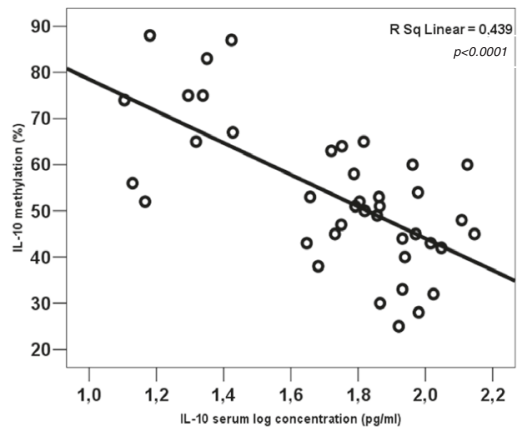

$\mathrm{B}$

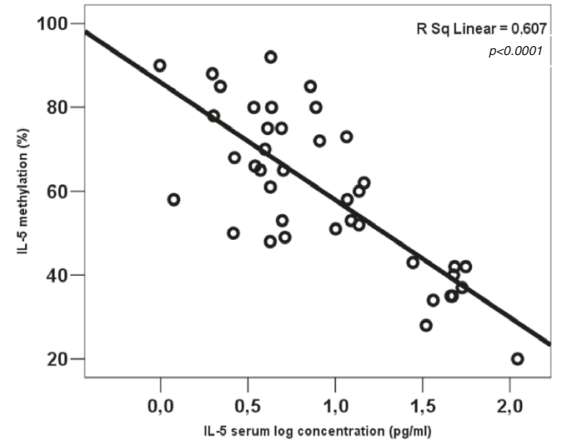

D

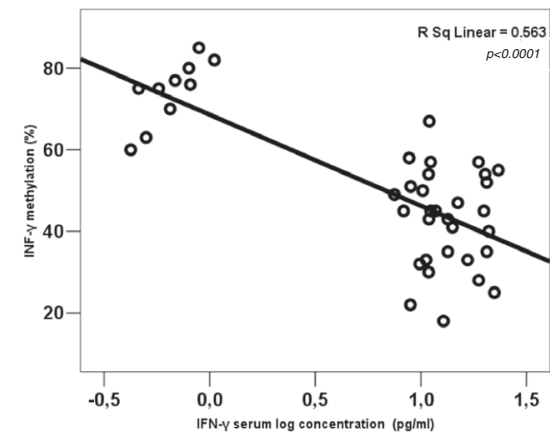

Figure 3 Correlation between IL-4, IL-5, IL-10, and INF- $\gamma$ gene DNA methylation rate and cytokine respective serum levels observed in groups 1, 2, and $\mathbf{3}$ (A-D). There is a negative and significant correlation between the DNA methylation rate and the respective serum concentration for all cytokines.

these differences, if confirmed in other studies, could be the basis for a potential use of these epigenetic marks as biomarkers of disease status.

In our study, the DNA methylation rate of the Th1 and Th2 cytokine genes differed significantly between CMA children treated with EHCF containing the probiotic Latobacillus rhamnosus GG, who acquired oral tolerance, and CMA children treated with other formulae. Among the factors potentially influencing DNA methylation rate in subjects who outgrew CMA, the strongest variable was EHCF + LGG treatment. This finding supports the use of these epigenetic marks as potential therapeutic biomarkers for dietary intervention. Interestingly, similar epigenetic effects have been reported for the farm-derived bacteria Acinetobacter lwoffli F78 in a mouse model of ovalbumin-induced asthma [28].

The main limitations of our study are the relative small number of children and cytokines evaluated and the cross-sectional design. Longitudinal cohort studies in CMA children from the onset of symptoms to the acquisition of oral tolerance are advocated. Another limitation is the PBMCs-based epigenetic evaluations. Given the small amounts of blood, as expected in a study of very young children, cell sorting was not possible. Although CMA is a systemic condition, for which the study of methylation patterns in whole blood may be feasible $[10,19]$, we cannot exclude the possibility that blood cell heterogeneity may have exerted a confounding effect.

\section{Conclusions}

While genetics may play a role in CMA, it cannot explain the rapid epidemiological changes occurred in recent decades. There is a growing recognition that the epigenome is a key missing piece of CMA pathogenesis puzzle [10]. Our findings suggest that DNA methylation plays a role in tuning the course of CMA and dietary interference with epigenetic mechanisms might represent an innovative approach to target the development of oral tolerance.

\section{Methods}

\section{Study population}

We planned to enroll two categories of IgE-mediated CMA patients (aged 3 to 18 months) consecutively referred to our tertiary Center for Pediatric Food Allergy because of the necessity of an oral food challenge: $i$. patients with recent evidence of IgE-mediated CMA ('active CMA patients', group 1); and ii. patients with a previous diagnosis of IgE-mediated CMA, but with 


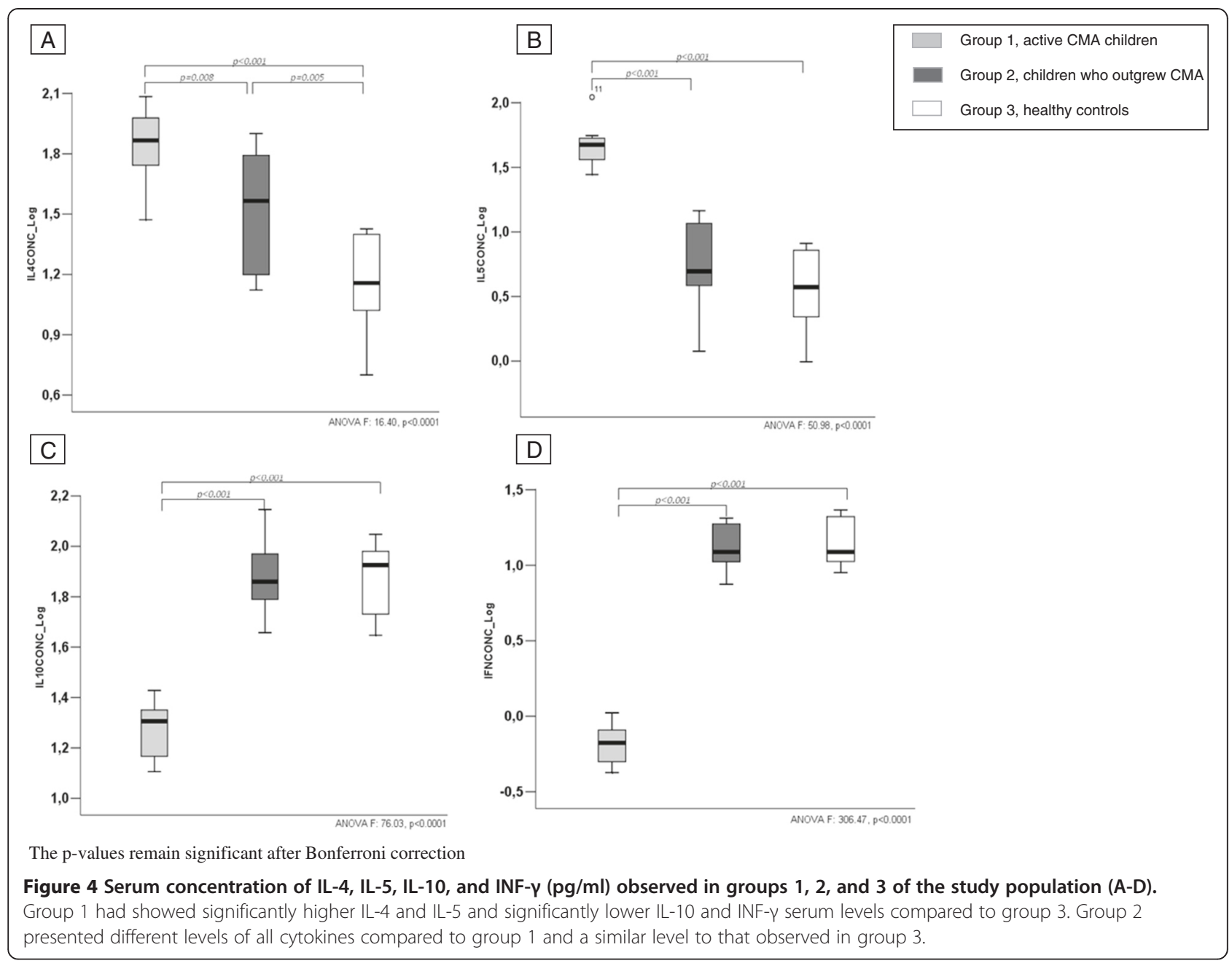

evidence of oral tolerance acquisition ('subjects who outgrew CMA', group 2).

In all patients, oral food challenge was requested to obtain diagnosis of CMA (group 1) or evidence of oral tolerance acquisition (group 2). The diagnosis of IgEmediated CMA was made in all subjects according to the result of a (DBPCFC, namely, occurrence of typical symptoms within $2 \mathrm{~h}$ of the administration of the last dose) and the outcomes of diagnostic work-up, that is, a clear clinical history of typical IgE-mediated symptoms of CMA, and positive specific serum IgE against proteins and epitopes of cow's milk [29]. Patients with a negative DBPCFC (group 2) were reassessed after 4 weeks to check the persistence of clinical tolerance. A venous blood sample $(4 \mathrm{ml})$ was obtained in all patients at the end of the DBPCFC procedure for serological test and epigenetic analysis. Th1 and Th2 cytokine dosage and PBMCs sorting were carried out with the same blood sample. Subjects with other allergic disorders or food allergies, eosinophilic disorders of the gastrointestinal tract, food protein-induced enterocolitis syndrome, concomitant chronic systemic diseases, congenital cardiac defects, active tuberculosis, autoimmune diseases, immunodeficiency, chronic inflammatory bowel diseases, celiac disease, cystic fibrosis, metabolic diseases, lactose intolerance, malignancy, chronic pulmonary diseases, and malformations of the gastrointestinal tract were excluded. As controls, during the same study period, consecutive healthy children, not at risk for atopic disorders (that is, without any first degree family member affected by atopic disorders), visiting our Center because of minimal surgical procedures were also enrolled (group 3). A venous blood sample $(4 \mathrm{ml})$ was collected from all these healthy subjects. These children were also assessed for the presence of food allergy and other allergic diseases at enrollment and 6 months after blood sampling by experienced pediatric allergists operating at the Center. The medical record of each child was recorded on a clinical chart. The study was approved by the Ethics Committee of the University of Naples 'Federico II' and was registered in Clinical Trials Protocol Registration System (ID number: NCT02062476). 


\section{Oral food challenge}

All food challenges were performed by DBPCFC, as previously described [30]. All oral food challenges took place at our Center on two separate days with a 1-week interval. Parents of children taking antihistamine were advised to withhold these medications for at least $72 \mathrm{~h}$ before and during the challenge. Randomization and preparation of the challenges were performed by experienced food allergy dieticians not directly involved in the procedures. Briefly, every $20 \mathrm{~min}$, successive doses $(0.1$, $0.3,1,3,10,30$, and $100 \mathrm{ml}$ ) of fresh pasteurized CM containing $3.5 \%$ fat or an amino acid-based formula were administered. Full emergency equipment and medications (epinephrine, antihistamines, and steroids) were available. The results were assessed simultaneously by three experienced pediatric allergists. Study subjects were scored for nine items divided into four main categories: $i$. General (lowered blood pressure plus tachycardia); ii. Skin (rash, urticaria/angioedema); iii. Gastrointestinal (nausea/ repeated vomiting, crampy-like abdominal pain, diarrhea); and $i v$. Respiratory (sneezing/itching, nasal congestion/ rhinorrhea, stridor deriving from upper airway obstruction or wheezing) on a 0 - to 3 -point scale ( 0 , none; 1 , light; 2 , moderate; and 3 , severe). If at least two of the three physicians independently scored any item at level 3, or 2 (or more) items at level 2, the test result was considered positive. Clinical symptoms occurring within $2 \mathrm{~h}$ of administering the highest dose were defined as 'IgE-mediated reactions.' The infants were observed for $2 \mathrm{~h}$ after the final dose, and then discharged. In the case of a positive DBPCFC, at any testing dose, the patient remained under observation until symptom resolution. If the patient did not show any symptom within the first $24 \mathrm{~h}$, parents were advised to give one single feed of $100 \mathrm{ml}$ of the tested formula (verum or placebo) everyday at home for 7 days. If any symptom occurred during this period, the patient returned to the outpatient clinic on the same day. After 7 days of verum or placebo administration, the patients were examined and the parents interviewed at the Center. To rule out a false-negative challenge result, parents were asked to contact the Center if any symptoms occurred in the 7 days after the DBPCFC procedures. The challenge was considered negative if the patient tolerated the entire challenge, including the observation period. Clinical tolerance acquisition was defined by the presence of a negative DBPCFC. The persistence of tolerance acquisition was checked after 4 weeks in all subjects with negative DBPCFC.

\section{Total IgE and specific lgE against proteins and epitopes of cow's milk}

Serum was obtained by centrifugation for 10 to $15 \mathrm{~min}$. Serum was flash frozen and stored at $-80^{\circ} \mathrm{C}$ until analysis. Serum total IgE and specific IgE against epitopes of cow's milk were analyzed by enzymatic immunoassay
(Phadia 100 ThermoFisher Scientific CAP system, Rodano Milano, Italy).

\section{Measurement of serum IL-4, IL-5, IL-10, and IFN- $\gamma$ concentration}

Serum samples were flash frozen and stored at $-80^{\circ} \mathrm{C}$ until further analysis. The concentrations of IL-4 and IL-10 were measured with a Human IL4/IL10 Enzyme immunoassay kit (Boster Biological Technology, Ltd., Fremont, CA, USA) according to the type of stimulation and stimulant. Human IL-5 and IFN- $\gamma$ ELISA, High Sensitivity (BioVendor, Brno, Czech Republic) were used to detect the IL-5 and IFN- $\gamma$ serum concentrations. Absorbance was read at $450 \mathrm{~nm}$. The minimum detection concentrations were $15.6 \mathrm{pg} / \mathrm{ml}$ for IL- $4,7.8 \mathrm{pg} / \mathrm{ml}$ for IL-5 and IL-10, and $0.78 \mathrm{pg} / \mathrm{ml}$ for IFN- $\gamma$.

\section{Leucocytes isolation, DNA extraction, and bisulfite modification}

PBMCs were isolated from whole blood samples using the Ficoll-Paque (GE Healthcare, Uppsala, Sweden) methods. DNA was extracted using the DNA Extraction Kit (GE Healthcare). One microgram of extracted DNA was modified with sodium bisulfite to convert all unmethylated, but not methylated-cytosines to uracil. Bisulfite conversion was carried out using the EZ DNA Methylation Gold Kit (ZYMO Research Co., Orange, CA, USA), according to the manufacturer's instructions. The converted DNA was stored at $-70^{\circ} \mathrm{C}$ until used. Fully methylated and fully unmethylated DNA (Merck Millipore, Darmstadt, Germany) were used as controls for the optimization of the assay conditions and to calculate the percent of methylation $(0 \%$ to $100 \%)$. The primer used for DNA methylation analysis of the promoter region of the IL-4, IL-5, IL-10, and IFN- $\gamma$ genes was designed in silico, using MethPrimer (http://www.urogene.org/methprimer/) (Table 2).

\section{High-resolution melting real-time PCR and sequencing}

Real-time PCR was performed with the LightCycler ${ }^{\circ} 480$ instrument (Roche Applied Science, Penzberg, Germany) using 96-well plates (Roche Applied Science). Extensive optimization experiments were performed in order to maximize PCR amplification efficiency, including PCR program parameters, $\mathrm{Mg}^{2+}$, primer and template concentrations. Sodium bisulfite-converted DNA (15 ng) was added to the PCR reaction mix, which consisted of highresolution melting Master Mix (Roche Applied Science), $0.25 \mu \mathrm{M}$ primers, and $\mathrm{Mg}^{2+}(2.5 \mathrm{mM}) \cdot \mathrm{dH}_{2} \mathrm{O}$ was used to supplement up to $20 \mu \mathrm{l}$. The real-time PCR protocol began with one cycle at $95^{\circ} \mathrm{C}$ for $10 \mathrm{~min}$ followed by 40 cycles of $95^{\circ} \mathrm{C}$ for $10 \mathrm{~s}, 61^{\circ} \mathrm{C}$ for $10 \mathrm{~s}$, and $72^{\circ} \mathrm{C}$ for $10 \mathrm{~s}$. Immediately after amplification, a re-annealing cycle consisting of $95^{\circ} \mathrm{C}$ for $1 \mathrm{~min}$ and a rapid cooling 
Table 2 The primer sequences used for high-resolution melting real-time PCR analysis

\begin{tabular}{|c|c|c|c|}
\hline Primers & Sequence $\left(5^{\prime}-3^{\prime}\right)$ & PCR product size (bp) & CpG sites $(n)$ \\
\hline IL-4 F & AGGTTAGGAGATGGAGATTATTTTG & 102 & 4 \\
\hline IL-4 R & TAAAACTACAAACACCTACCACCAC & & \\
\hline IL-5 F & AGGTTAGGAGATGGAGATTATTTG & 102 & 5 \\
\hline IL-5 R & TAAAACTACAAACACCTACCACCAC & & \\
\hline $\mathrm{IFN}-\mathrm{F}$ & GAGTITGTITGTTATTAGGTTGG & 124 & 6 \\
\hline IFN- $\gamma R$ & AATACCTATAATCCCAACTACTC & & \\
\hline IL-10 F & TTATTGTGTTAATTAGGATGGTT & 154 & 7 \\
\hline IL-10 R & TAAACAAAAATCCAAAATACTTITT & & \\
\hline
\end{tabular}

to $65^{\circ} \mathrm{C}$ for $1 \mathrm{~min}$ was introduced to prepare the melting curve acquisition step. Real-time fluorescence acquisition was set at the elongation step $\left(72^{\circ} \mathrm{C}\right)$. Samples whose amplification begun late and sample whose relative fluorescence value on the raw melting-curve plot was low were not further processed. All PCR reactions were performed in triplicate for each sample. Melting data acquisition began at $69^{\circ} \mathrm{C}$ and ended in $95^{\circ} \mathrm{C}$, using a ramp rate of $0.2^{\circ} \mathrm{C} / \mathrm{s}$. High-resolution melting analysis was also performed with the LightCycler ${ }^{\oplus} 480$ instrument (Roche Applied Science) using 96-well plates (Roche Applied Science). Data processing included normalization and resulted on the normalized melting curves and the respective negative derivative of fluorescence over the temperature plots, using the LightCycler $480^{\circ}$ gene scanning software. The settings for data collection were 50 fluorescence acquisition points per degree centigrade resulting on a ramp rate of $0.01^{\circ} \mathrm{C} / \mathrm{s}$. Comparison of the melting curve or the peaks of an unknown sample with those of the controls gave the semi-quantitative estimation for the methylation level of that sample. The results were confirmed by direct sequencing (Sanger method modified: ddNTPs labeled with four different fluorophores) and analyzed by capillary electrophoresis (analytical specificity and sensitivity of the test: $>99 \%$ ).

\section{Statistical analysis}

The Kolmogorov-Smirnov test was used to determine whether variables (cytokine DNA methylation rates and serum levels of cytokines and IgE) were normally distributed. Serum levels of cytokines and IgE were not normally distributed (Kolmogorov-Smirnov test; $P<0.05$ ) and thus were 10 log-transformed (Kolmogorov-Smirnov test; $P>0.05)$.

The $\chi 2$ test and Fisher's exact test were used for categorical variables. To evaluate the differences among continuous variables, one-way ANOVA and the $t$-test were performed. To determine which groups in the sample differ, the Bonferroni correction was performed. Pearson's correlation coefficient ' $r$ ' was used to evaluate the correlation between continuous variables.
Given the small sample size and relative issues of separability, a backward stepwise Firth's bias reduced logistic regression was used to identify the best predictor of the CMA disease state [31]. This analysis was performed with the $\mathrm{R}$ package 'logistf'. Linear regression was used to estimate the relationship between continuous variables. The level of significance for all statistical tests was two-sided, $P<0.05$. All data were recorded in a dedicated database and analyzed by a statistician, blinded to the group assignment of patients, with SPSS software (SPSS Inc, version 21.0, Chicago, IL, USA).

\section{Abbreviations \\ AAF: amino acid-based formula; APT: atopy patch testing; CMA: cow's milk allergy; DBPCFC: double blind placebo controlled food challenge; EHCF: extensively hydrolyzed casein formula; EHCF + LGG: extensively hydrolyzed casein formula plus Lactobacillus GG; IgE: immunoglobulin E; IL-10: interleukin-10; IL-4: interleukin-4; IL-5: interleukin-5; INF- $\gamma$ : interferon- $\gamma$; LGG: Lactobacillus rhamnosus GG; PBMCs: peripheral blood mononuclear cells; RHF: hydrolyzed rice formula; SF: soy formula; SPT: skin prick testing.}

\section{Competing interests}

The authors declare that they have no competing interests.

\section{Authors' contributions}

RBC, LP, RN, and FS designed the study, coordinated the research team, and wrote the first draft of the report. $R N, C L, P V$, and DCM cared for the patients and LP, DMV and DV performed laboratory activities. MC and LG performed statistical analysis and interpretation of data. All authors revised and approved the final version of the article.

\section{Acknowledgements}

This work is supported in part by the Italian Ministry of Health Grant PE-201102348447, and by unrestricted grant from Mead Johnson Nutrition (Evansville, Indiana, USA) devoted to the University of Naples 'Federico II.' However, the Italian Ministry of Health and Mead Johnson Nutrition have no influence on: (1) the study design, (2) the collection, analysis, and interpretation of data; (3) the writing of the manuscript; and 4) the decision to submit the manuscript for publication. The authors thank Jean Ann Gilder (Scientific Communication srl., Naples, Italy) for editing the text.

\section{Author details}

${ }^{1}$ Department of Translational Medical Science, University of Naples 'Federico II', Via S. Pansini, 580131 Naples, Italy. ${ }^{2}$ European Laboratory for the Investigation of Food-Induced Diseases, University of Naples 'Federico II', Via S. Pansini, 580131 Naples, Italy. ${ }^{3}$ Department of Molecular Medicine and Medical Biotechnologies, University of Naples Federico I, Via S.Pansini, 5 80131 Naples, Italy. ${ }^{4}$ CEINGE-Biotecnologie Avanzate s.c.ar.l, University of Naples 'Federico II', Via S.Pansini, 580131 Naples, Italy. 
Received: 22 December 2014 Accepted: 10 March 2015

Published online: 31 March 2015

\section{References}

1. Høst A. Frequency of cow's milk allergy in childhood. Ann Allergy Asthma Immunol. 2002;89(6 Suppl 1):33-7.

2. Sicherer SH, Sampson HA. Food allergy: epidemiology, pathogenesis, diagnosis, and treatment. J Allergy Clin Immunol. 2014;133:291-307. quiz 308.

3. Høst A, Halken S, Jacobsen HP, Christensen AE, Herskind AM, Plesner K. Clinical course of cow's milk protein allergy/intolerance and atopic diseases in childhood. Pediatr Allergy Immunol. 2002;13 Suppl 15:23-8.

4. Oyoshi MK, Oettgen HC, Chatila TA, Geha RS, Bryce PJ. Food allergy: insights into etiology, prevention, and treatment provided by murine models. J Allergy Clin Immunol. 2014;133:309-17.

5. Vocca I, Berni Canani R, Camarca A, Ruotolo S, Nocerino R, Radano G, et al. Peripheral blood immune response elicited by beta-lactoglobulin in childhood cow's milk allergy. Pediatr Res. 2011;70:549-54

6. Gunawardhana LP, Gibson PG, Simpson JL, Benton MC, Lea RA, Baines KJ. Characteristic DNA methylation profiles in peripheral blood monocytes are associated with inflammatory phenotypes of asthma. Epigenetics. 2014:9:1302-16

7. Kuo CH, Hsieh CC, Lee MS, Chang KT, Kuo HF, Hung CH. Epigenetic regulation in allergic diseases and related studies. Asia PacAllergy. 2014:4:14-8.

8. Berger SL, Kouzarides T, Shiekhattar R, Shilatifard A. An operational definition of epigenetics. Genes Dev. 2009;23:781-3.

9. Kabesch M, Michel S, Tost J. Epigenetic mechanisms and the relationship to childhood asthma. Eur Respir J. 2010;36:950-61.

10. Hong $X$, Wang $X$. Epigenetics and development of food allergy (FA) in early childhood. Curr Allergy Asthma Rep. 2014;14:460.

11. van Panhuys N, Le Gros G, McConnell MJ. Epigenetic regulation of Th2 cytokine expression in atopic diseases. Tissue Antigens. 2008;72:91-7.

12. Lee DU, Agarwal S, Rao A. Th2 lineage commitment and efficient IL-4 production involves extended demethylation of the IL-4 gene. Immunity. 2002;16:649-60.

13. Tykocinski LO, Hajkova P, Chang HD, Stamm T, Sozeri O, Lohning M, et al. A critical control element for interleukin-4 memory expression in Thelper lymphocytes. J Biol Chem. 2005;280:28177-85.

14. Wei G, Wei L, Zhu J, Zang C, Hu-Li J, Yao Z, et al. Global mapping of $\mathrm{H} 3 \mathrm{~K} 4 \mathrm{me} 3$ and $\mathrm{H} 3 \mathrm{~K} 27 \mathrm{me} 3$ reveals specificity and plasticity in lineage fate determination of differentiating CD4+ T cells. Immunity. 2009:30:155-67.

15. Liu J, Bellaney M, Al-alem U, Quan C, Jin X, Perera F, et al. Combined inhaled diesel exhaust particles and allergen exposure alter methylation of $\mathrm{T}$ helper genes and IgE production in vivo. Tox Sci. 2008;102:76-81.

16. Torrone D, Kuriakose J, Moors K, Jiang H, Niedzwiecki M, Perera F, et al. Reproducibility and intraindividual variation over days in buccal cell DNA methylation of two asthma genes, interferon gamma (IFNgamma) and inducible nitric oxide synthase (iNOS). Clin Epigenetics. 2012;4:3.

17. Bjornsson HT, Sigurdsson MI, Fallin MD, Irizarry RA, Aspelund T, Cui H, et al. Intra-individual change over time in DNA methylation with familial clustering. JAMA. 2008;299:2877-83.

18. Talens RP, Christensen K, Putter H, Willemsen G, Christiansen L, Kremer D, et al. Epigenetic variation during the adult lifespan: cross-sectional and longitudinal data on monozygotic twin pairs. Aging Cell. 2012;11:694-703.

19. Kabesch M. Epigenetics in asthma and allergy. Curr Opin Allergy Clin Immunol. 2014;14:62-8.

20. Jones B, Chen J. Inhibition of IFN-gamma transcription by site-specific methylation during T helper cell development. EMBO J. 2006;25:2443-52.

21. Dong J, Chang HD, Ivascu C, Qian Y, Rezai S, Okhrimenko A, et al. Loss of methylation at the IFNG promoter and CNS-1 is associated with the development of functional IFN-gamma memory in human CD4(+) T lymphocytes. Eur J Immunol. 2013;43:793-804.

22. Kwon NH, Kim JS, Lee JY, Oh MJ, Choi DC. DNA methylation and the expression of IL-4 and IFN-gamma promoter genes in patients with bronchial asthma. J Clin Immunol. 2008;28:139-46.

23. Lovinsky-Desir S, Ridder R, Torrone D, Maher C, Narula S, Scheuerman M, et al. Methylation of the allergy regulatory gene interferon gamma varies by age, sex, and tissue type in asthmatics. Clin Epigenetics. 2014;6:9.
24. Kohli A, Garcia MA, Miller RL, Maher C, Humblet O, Hammond SK, et al. Secondhand smoke in combination with ambient air pollution exposure is associated with increased CPG methylation and decreased expression of IFN-gamma in T effector cells and Foxp3 in T regulatory cells in children. Clin Epigenetics. 2012;4:17.

25. Martino D, Joo JE, Sexton-Oates A, Dang T, Allen K, Saffery $R$, et al. Epigenome-wide association study reveals longitudinally stable DNA methylation differences in CD4+ T cells from children with IgE-mediated food allergy. Epigenetics. 2014;9:998-1006.

26. Suárez-Álvarez B, Baragaño Raneros A, Ortega F, López-Larrea C. Epigenetic modulation of the immune function: a potential target for tolerance. Epigenetics. 2013:8:694-70.

27. Syed A, Garcia MA, Lyu SC, Bucayu R, Kohli A, Ishida S, et al. Peanut oral immunotherapy results in increased antigen-induced regulatory T-cell function and hypomethylation of forkhead box protein 3 (FOXP3). J Allergy Clin Immunol. 2014;133:500-10.

28. Brand S, Teich R, Dicke T, Harb H, Yildirim AÖ, Tost J, et al. Epigenetic regulation in murine offspring as a novel mechanism for transmaternal asthma protection induced by microbes. J Allergy Clin Immunol. 2011;128:618.

29. Berni Canani R, Nocerino R, Terrin G, Coruzzo A, Cosenza L, Leone L, et al. Effect of Lactobacillus GG on tolerance acquisition in infants with cow's milk allergy: a randomized trial. J Allergy Clin Immunol. 2012;129(2):580-2, 582.e1-5.

30. Berni Canani R, Nocerino R, Terrin G, Frediani T, Lucarelli S, Cosenza L, et al. Formula selection for management of children with cow's milk allergy influences the rate of acquisition of tolerance: a prospective multicenter study. J Pediatr. 2013;163:771-7.e1.

31. Firth D. Bias reduction of maximum likelihood estimates. Biometrika. 1993:80:27-38

\section{Submit your next manuscript to BioMed Central and take full advantage of:}

- Convenient online submission

- Thorough peer review

- No space constraints or color figure charges

- Immediate publication on acceptance

- Inclusion in PubMed, CAS, Scopus and Google Scholar

- Research which is freely available for redistribution 